Journal for ... ImmunoTherapy of Cancer

\section{Efficient elimination of primary B-ALL cells in vitro and in vivo using a novel 4-1BB-based CAR targeting a membrane-distal CD22 epitope}

To cite: Velasco-Hernandez $\mathrm{T}$, Zanetti SR, Roca-Ho H, et al. Efficient elimination of primary B-ALL cells in vitro and in vivo using a novel 4-1BB-based CAR targeting a membranedistal CD22 epitope. Journal for ImmunoTherapy of Cancer 2020;8:e000896. doi:10.1136/ jitc-2020-000896

- Additional material is published online only. To view please visit the journal online (http://dx.doi.org/10.1136/jitc2020-000896).

Accepted 22 June 2020

Check for updates

(c) Author(s) (or their employer(s)) 2020. Re-use permitted under CC BY-NC. No commercial re-use. See rights and permissions. Published by BMJ.

For numbered affiliations see end of article.

\section{Correspondence to}

Dr Talia Velasco-Hernandez; tvelasco@carrerasresearch.org

Dr Pablo Menendez; pmenendez@carrerasresearch. org

\section{ABSTRACT}

Background There are few therapeutic options available for patients with B-cell acute lymphoblastic leukemia (B-ALL) relapsing as $\mathrm{CD}^{-} 9^{-}$either after chemotherapy or CD19targeted immunotherapies. CD22-chimeric antigen receptor (CAR) T cells represent an attractive addition to CD19-CAR T cell therapy because they will target both $\mathrm{CD}_{22}{ }^{+} \mathrm{CD} 19^{-}$ B-ALL relapses and CD19- preleukemic cells. However, the immune escape mechanisms from CD22-CAR T cells, and the potential contribution of the epitope binding of the anti-CD22 single-chain variable fragment (scFv) remain understudied.

Methods Here, we have developed and comprehensively characterized a novel CD22-CAR (clone hCD22.7) targeting a membrane-distal CD22 epitope and tested its cytotoxic effects against B-ALL cells both in in vitro and in vivo assays.

Results Conformational epitope mapping, cross-blocking, and molecular docking assays revealed that the hCD22.7 $\mathrm{scFv}$ is a high-affinity binding antibody which specifically binds to the ESTKDGKVP sequence, located in the lg-like V-type domain, the most distal domain of CD22. We observed efficient killing of B-ALL cells in vitro, although the kinetics were dependent on the level of CD22 expression. Importantly, we show an efficient in vivo control of patients with B-ALL derived xenografts with diverse aggressiveness, coupled to long-term hCD22.7-CAR T cell persistence. Remaining leukemic cells at sacrifice maintained full expression of CD22, ruling out CAR pressure-mediated antigen loss. Finally, the immunogenicity capacity of this hCD22.7-scFv was very similar to that of other CD22 scFv previously used in adoptive T cell therapy.

Conclusions We report a novel, high-affinity hCD22.7 scFv which targets a membrane-distal epitope of CD22. 4-1BBbased hCD22.7-CAR T cells efficiently eliminate clinically relevant $\mathrm{B}-\mathrm{CD} 22^{\text {high }}$ and $\mathrm{CD} 22^{\text {low }} \mathrm{ALL}$ primary samples in vitro and in vivo. Our study supports the clinical translation of this hCD22.7-CAR as either single or tandem CD22-CD19CAR for both naive and anti-CD19-resistant patients with B-ALL.

\section{BACKGROUND}

B-cell acute lymphoblastic leukemia (B-ALL) is an aggressive cancer, diagnosed at any age throughout the lifespan of an individual, being the most common malignancy in children. ${ }^{1}$ Despite current 5-year diseasefree survival rates of $\sim 80 \%$, refractory and relapsed $(\mathrm{R} / \mathrm{R})$ patients have a dismal prognosis. $^{2-7}$ Adult B-ALL is less frequent, but is commonly associated with an unfavorable clinical outcome even after the use of highdose chemotherapy regimens and allogenic stem cell transplantation. ${ }^{8}$

Immunotherapy has generated unprecedented expectations in cancer treatment. Adoptive cellular immunotherapy, based on the genetic engineering of human $\mathrm{T}$ cells with chimeric antigen receptors (CARs) redirected against cell surface tumor antigens, has shown great potential in $R / R$ B-ALL due to the high efficacy and specificity of CD19-CAR T cells, with $\sim 90 \%$ response rates and $\sim 50 \%$ complete remission rates after 12 months. ${ }^{9} 10$ However, CD19-CAR T cells are not always curative and $\sim 60 \%$ of patients inevitably relapse, mainly because of poor CAR $T$ cell persistence or the emergence of CD19- B-ALL clones. ${ }^{11} 12$ Of note, a very recent and elegant clinical study has shown that a low-affinity CD19-CAR enhances CAR $\mathrm{T}$ cell expansion and persistence in patients with B-ALL, but it is associated with immune escape and an increased number of CD19relapses. ${ }^{12}$ Unfortunately, there are very few therapeutic options for patients with B-ALL relapsing as $\mathrm{CD}^{-} 9^{-}$after either chemotherapy or CD19-directed immunotherapy and, therefore, novel strategies are being explored to enhance CAR T cell function and persistence, 
including the development of novel target antigens, costimulatory domains or effector cells. ${ }^{13}$

Like CD19, CD22 is expressed in a B-cell lineagerestricted fashion. ${ }^{14} \mathrm{CD} 22$-directed $\mathrm{T}$ cells are an attractive addition to the current $\mathrm{CD} 19$-directed $\mathrm{T}$ cell strategies because (1) they constitute an alternative for $\mathrm{CD} 19^{-/ \mathrm{dim}}$ B-ALL relapses as CD22 is retained in such relapses ${ }^{15}$ and (2) they would target the earliest pre-VDJ CD19-B-lineage progenitors, which potentially can escape in CD19-CAR $\mathrm{T}$ cell therapy. ${ }^{16}$ Indeed, CD22 has been employed as a target for B-ALL in clinical trials using anti-CD22 monoclonal antibodies (moAb) linked to immunotoxins, ${ }^{17-19}$ and several CD22-CARs have also been reported. ${ }^{15}{ }^{20-22}$ CD22-CAR T cells induce clinical remission in $\sim 70 \%$ of patients with R/R B-ALL either naive or resistant to CD19-CAR T cells. ${ }^{15} 2123$ Moreover, these studies reported somewhat different clinical results regarding the potential mechanism of immune escape of B-ALL blasts from CD22-CAR T cells-mediated lysis with subsequent CD22dim relapses. ${ }^{15}{ }^{21}$ Importantly, comparative studies from the Orentas laboratory suggested that maximal efficacy of CD22-CAR T cells is obtained when using anti-CD22 antibodies that target cell surface proximal epitopes or that display a high-binding affinity for CD22. ${ }^{20}$ Here, we have developed and characterized a novel high-affinity CD22-CAR targeting a membrane-distal CD22 epitope, and report an efficient elimination in vitro and in vivo of clinically relevant primary B-ALL cells.

\section{MATERIALS AND METHODS \\ Generation of a CD22 single-chain variable fragment (scFv)}

MoAbs reactive with human CD22 were generated by fusion of NS-1 myeloma cells with splenocytes from a Balb/c mouse previously immunized three times with $30 \times 10^{6}$ CD22-transfected 300.19 cells. Supernatants from hybridoma-containing wells were screened by flow cytometry for moAbs reactive with CD22-transfected 300.19 cells. Three reactive hybridomas were chosen for further characterization and were subcloned by limiting dilution (clones hCD22.7, hCD22.316 and hCD22.401). The three antibodies were IgG1 $\kappa$ isotype, as determined using a mouse moAb isotyping kit (Boehringer Mannheim, Mannheim, Germany). Antibody specificity was further validated using a CD22-transfected COS cell line (pUNO1-hCD22; InvivoGen, Toulouse, France), the cell lines Raji, Daudi, PRMI 82229, U266 and Jurkat, and peripheral blood (PB) mononuclear cells (PBMCs). CD22 epitope binding to the hCD22.7 clone was determined by cross-blocking ${ }^{24}$ with an antibody against the N-terminal domain of CD22 (S-HCL-1). ${ }^{25}$ The CD22-specific scFv derived from the hCD22.7 clone was obtained using the mouse IgG Library Primer Set (Progen, Heidelberg, Germany).

\section{Immunogenicity prediction}

Immunogenicity potential was predicted using the in silico tool NetMHC V.4.0. ${ }^{26}$ Analyzed scFvs were broken in 9-mer peptides, length for which human leukocyte antigen (HLA) molecules have a strong preference. Peptides were considered to bind major histocompatibility complex class I (MHC-I) when a half-maximum inhibitory concentration $\left(\mathrm{IC}_{50}\right)$ is $<500 \mathrm{nM}$, being considered strong binders when $\mathrm{IC}_{50}<50 \mathrm{nM}$. Only HLA supertype representatives were analyzed. The predicted epitope-binding affinity was calculated as $1 / \mathrm{IC}_{50} 100 .^{28}$

\section{Epitope mapping}

Reactivity of the hCD22.7 moAb was subjected to PEPperMAP Conformational Epitope Mapping against CD22 translated into cyclic constrained peptides (PEPperPRINT GmbH, Heidelberg, Germany). The sequence of CD22 (UniProtKB P20273) was elongated with neutral GSGSGSG linkers at the C-terminus and $\mathrm{N}$-terminus to avoid truncated peptides. The elongated antigen sequence was translated into 7, 10 and 13 amino acid peptides with a peptide-peptide overlap of 6,9 and 12 amino acids. After peptide synthesis, all peptides were cyclized via a thioether linkage between a C-terminal cysteine and an appropriately modified N-terminus. The resulting conformational CD22 peptide microarrays contained 2556 different peptides printed in duplicate (5112 peptide spots), and were framed by additional human influenza hemagglutinin (HA, YPYDVPDYAG, 146 spots) control peptides. Hibridoma supernatant containing hCD22.7 moAb was diluted in incubation buffer (phosphate-buffered saline (PBS), $\mathrm{pH}$ 7.4, with $0.005 \%$ Tween 20) and 10\% MB-070 blocking buffer (Rockland Immunochemicals, Limerick, Pennsylvania, USA) and incubated with peptide microarrays for 16 hours at $4^{\circ} \mathrm{C}$ and swirling at $140 \mathrm{rpm}$. Unbound antibodies were removed by washing the arrays with washing buffer (PBS, pH 7.4, with 0.005\% Tween 20). Prestaining of the peptide arrays was done with $0.2 \mu \mathrm{g} / \mathrm{mL}$ goat antimouse IgG $(\mathrm{H}+\mathrm{L})$ antibody conjugated to DyLight680 (Thermo Fisher Scientific). HA control peptides framing the peptide arrays were finally stained with $0.5 \mu \mathrm{g} / \mathrm{mL}$ monoclonal anti-HA (12CA5)-DyLight800 (Thermo Fisher Scientific) for $45 \mathrm{~min}$ at room temperature (RT). Quantification of spot intensities and peptide annotation was performed with the LI-COR Odyssey Imaging System (scanning offset $0.65 \mathrm{~mm}$, resolution $21 \mu \mathrm{m}$, and scanning intensities of $7 / 7($ red $=700 \mathrm{~nm} /$ green $=800 \mathrm{~nm})$ and PepSlide Analyzer.

\section{Structural model}

The structural model of the CD22-hCD22.7-scFv complex was obtained using directed docking as follows. The structure for CD22 was taken for the PDB databank ${ }^{29}$ (identification code $5 \mathrm{vkj}^{30}$ ) and the structure of the scFv anti-CD22 was derived by homology modeling using M4T ${ }^{31}$ and templates $4 \mathrm{~h} 0 \mathrm{~h},{ }^{32} 1 \mathrm{dqd}^{33}$ and $4 \mathrm{okv}^{34}$ The initial docking conformations were inferred using PatchDock $^{35}$ by selecting the complementarity-determining regions of anti-CD22-scFv and mapped epitope of CD22 as docking interfaces. Resulting docking conformations 
were further refined and minimized using Rosetta ${ }^{36}$ and the top 200 poses were visually inspected. A more detailed description of the structural analysis is shown in online supplementary information.

\section{CAR design and vectors, lentiviral production and T cells transduction}

The CD22-specific scFv was cloned into a pCCL lentiviralbased backbone containing a human CD8 hinge and transmembrane (TM) domain, human 4-1BB and CD3 $\zeta$ endodomains (second-generation CAR), and a T2A-GFP cassette. $^{37}$ An identical lentiviral vector with the CD8 TM-4-1BB-CD3 $\zeta$ domains linked to a His-Tag was used as a mock intracellular (mock-IC) control. CAR-expressing viral particles pseudotyped with VSV-G were generated by cotransfection of HEK $293 \mathrm{~T}$ cells with the pCCL vector and the packaging plasmids VSV-G and psPAX2 using polyethylenimine (PEI, Polysciences, Warrington, Pennsylvania, USA). Supernatants were collected at 48 and 72 hours after transfection and concentrated by ultracentrifugation.

PBMCs were isolated from buffy coats from healthy volunteers by Ficoll-Hypaque gradient centrifugation (GE Healthcare, Chicago, Illinois, USA). Buffy coats were obtained from the Barcelona Blood and Tissue Bank on Institutional Review Board approval (HCB/2018/0030). $\mathrm{T}$ cells were activated by plate coating with anti-CD3 (OKT3) and anti-CD28 (CD28.2) antibodies (BD Biosciences, Franklin Lakes, New Jersey, USA) for 2 days and were transduced with the CAR-expressing lentivirus at a multiplicity of infection of 10 in the presence of interleukin 7 (IL-7) and IL-15 (10 ng/mL; Miltenyi Biotec, Bergisch Gladbach, Germany). ${ }^{37} \mathrm{~T}$ cells were expanded in RPMI-1640 medium (Gibco/Invitrogen, Waltham, MA) containing $10 \%$ heat-inactivated fetal bovine serum (FBS, Sigma, St. Louis, Missouri, USA), penicillinstreptomycin (Gibco/Invitrogen), and IL-7 and IL-15 $(10 \mathrm{ng} / \mathrm{mL})$, for up to 10 days. Surface expression of CD22-CAR was traced by fluorescence-activated cell sorting (FACS).

\section{Cell lines}

SEM, NALM6, MV4-11 and REH cells lines were purchased from the DSMZ cell line bank (Braunschweig, Germany). CD19-knock out (KO) and CD22-KO SEM cells were generated by CRISPR-mediated genome editing. Briefly, 200,000 cells were electroporated (Neon transfector, ThermoFisher Scientific, Waltham, Massachusetts, USA) with a Cas9 protein/tracrRNA/ crRNA complex (IDT, Coralville, Iowa, USA). Two guides were designed for each gene: CD19-exon 2.1, CAGGCCTGGGAATCCACATG and CD19-exon 14.1, AGAACATGGATAATCCCGAT; and CD22-exon 3.2, TCAATGACAGTGGTCAGCTG and CD22-exon 9, CAGGTGTAGTGGGAGACGGG. After electroporation, cells were allowed to recover and then $\mathrm{CD} 19^{-}$or $\mathrm{CD}_{2}{ }^{-}$ cells were FACS sorted ( $>99 \%$ purity).

\section{In vitro cytotoxicity assays and cytokine release determination}

Target cells (cell lines or primary B-ALL cells; 100,000 target cells/well of a 96-well plate) were incubated with CD22-CAR or mock-IC T cells at different effector:target (E:T) ratios for the indicated time periods. Cell lines were cultured in RPMI-1640, 10\% FBS and penicillin-streptomycin. Primary cells were cultured in StemSpan ${ }^{\mathrm{TM}}$ SFEM media (StemCell Technologies, Vancouver, Canada), 20\% FBS, penicillin-streptomycin, insulin-transferrin-selenium (Gibco/Invitrogen), hSCF (100 ng/mL), hFLT3L (100 ng/mL), hIL3 (10 ng/mL) and hIL7 (10 ng/mL, all from Miltenyi Biotec, Bergisch Gladbach, Germany). CAR T cell-mediated cytotoxicity was determined by analyzing the residual live (7-aminoactinomycin $\mathrm{D}^{-} ; 7$ - $\mathrm{AAD}^{-}$) target cells at each time point and E:T ratio. For absolute cell counting, BD TruCount ${ }^{\mathrm{TM}}$ absolute count tubes (BD Biosciences) were used. Quantification of the proinflammatory cytokines IL2, TNF $\alpha$ and IFN $\gamma$ was measured by ELISA using BD OptEIA ${ }^{\mathrm{TM}}$ Human ELISA kits (BD Biosciences), in supernatants harvested at 24 hours (cell lines) and 48 hours (primary cells) post $\mathrm{T}$ cell-exposure, using an 1:1 E:T ratio. Online supplementary table 1 shows clinic-biological features of the B-ALL samples used.

\section{Flow cytometry}

Cell surface expression of the CD22-CAR was confirmed by green fluorescence protein (GFP) expression, binding to an AffiniPure $\mathrm{F}\left(\mathrm{ab}^{\prime}\right)_{2}$ fragment goat antimouse IgG (H+L)-APC (Jackson ImmunoResearch, Westgrove, Pennsylvania, USA), and an anti-His-APC (J095G46; BioLegend, San Diego, California, USA), after prior incubation with human recombinant CD22-His (rCD22-His, ThermoFisher Scientific). Activation of transduced $\mathrm{T}$ cells was confirmed 48 hours after plating of PBMCs by surface staining with CD3-PE (UCHT1; BD Biosciences), CD25-APC (M-A251; BD Biosciences) and CD69-VioBlue (REA824; Miltenyi Biotec). T cells and target cells were identified in in vitro assays using the following human antibodies: CD3-PE (UCHT1), CD22-APC (HIB22), CD19-BV421 (HIB19) and CD10PECy7 (HI10a) or CD13-PECy7 (WM15, for SEM cells) or CD33-APC (WM53, for MV4-11 cells, BD Biosciences). Dead cells were discarded by 7AAD staining. Cells collected from mouse $\mathrm{PB}$, bone marrow (BM) and spleen were stained with HLA-ABC-PE (G46-2.6), CD45BV510 (HI30), CD3-PerCP (SK7), CD22-APC, CD19BV421 and CD10-PECy7 (BD Biosciences). Cells were incubated for $30 \mathrm{~min}$ and then lysed and fixed with the BD FACS ${ }^{\mathrm{TM}}$ Lysing solution (BD Biosciences). Fluorescence Minus One controls were used to set the gates (online supplementary figure 1). A FACSCanto ${ }^{\mathrm{TM}}$-II flow cytometer and a FACSDiva ${ }^{\mathrm{TM}}$ software was used for the analysis (BD Biosciences). 


\section{In vivo xenograft models for B-ALL and CAR T cells}

Twelve-week-old non-obese diabetic (NOD).Cg-Prk$\mathrm{dc}^{\text {scid }} \mathrm{Il} \mathrm{rg}^{\mathrm{tm} 1 \mathrm{Wjl}} / \mathrm{SzJ}$ (NSG) mice (The Jackson Laboratory, Bar Harbor, Maine USA) were bred and housed under pathogen-free conditions. All in vivo procedures were approved by a local ethics committee (HRH-17$0029 \mathrm{P} 1)$. In total, $0.5-1.5 \times 10^{6}$ patient derived xenograft (PDX) B-ALL cells were intra-BM transplanted in sublethally irradiated (2 Gy) NSG mice, followed by intravenous infusion of $4 \times 10^{6} \mathrm{CD} 22$-CAR T cells (average transduction $\sim 51.3 \%$ ) or mock-IC T cells (average transduction $\sim 54.2 \%) 2$ weeks later. B-ALL engraftment was monitored in PB every other week. BM aspirates were analyzed 6 weeks after transplantation and at sacrifice, along with spleens. Red blood cell counts were determined with a hemocytometer 2800VET V-Sight (Menarini Diagnostics, Badalona, Spain).

\section{Statistical analysis}

All data are expressed as the mean \pm SEM. Differences between groups were assessed using two-tailed unpaired Student's t-tests, or paired Student's t-tests when analyzing data from different donors, unless otherway stated. All analyses were performed with Prism software, V.8.0 (GraphPad software, San Diego, California, USA).

\section{RESULTS}

hCD22.7-CAR T cells efficiently eliminate B-ALL cells in vitro Our novel anti-CD22 scFv (clone hCD22.7) was cloned into a second-generation CAR construct consisting of an anti-CD22 scFv, a CD8 hinge/TM spacer, and intracellular signaling domains from $4-1 \mathrm{BB}$ and $\mathrm{CD} 3 \zeta$, coupled in-frame with GFP through a T2A sequence (figure 1A). To identify the domain of CD22 recognized
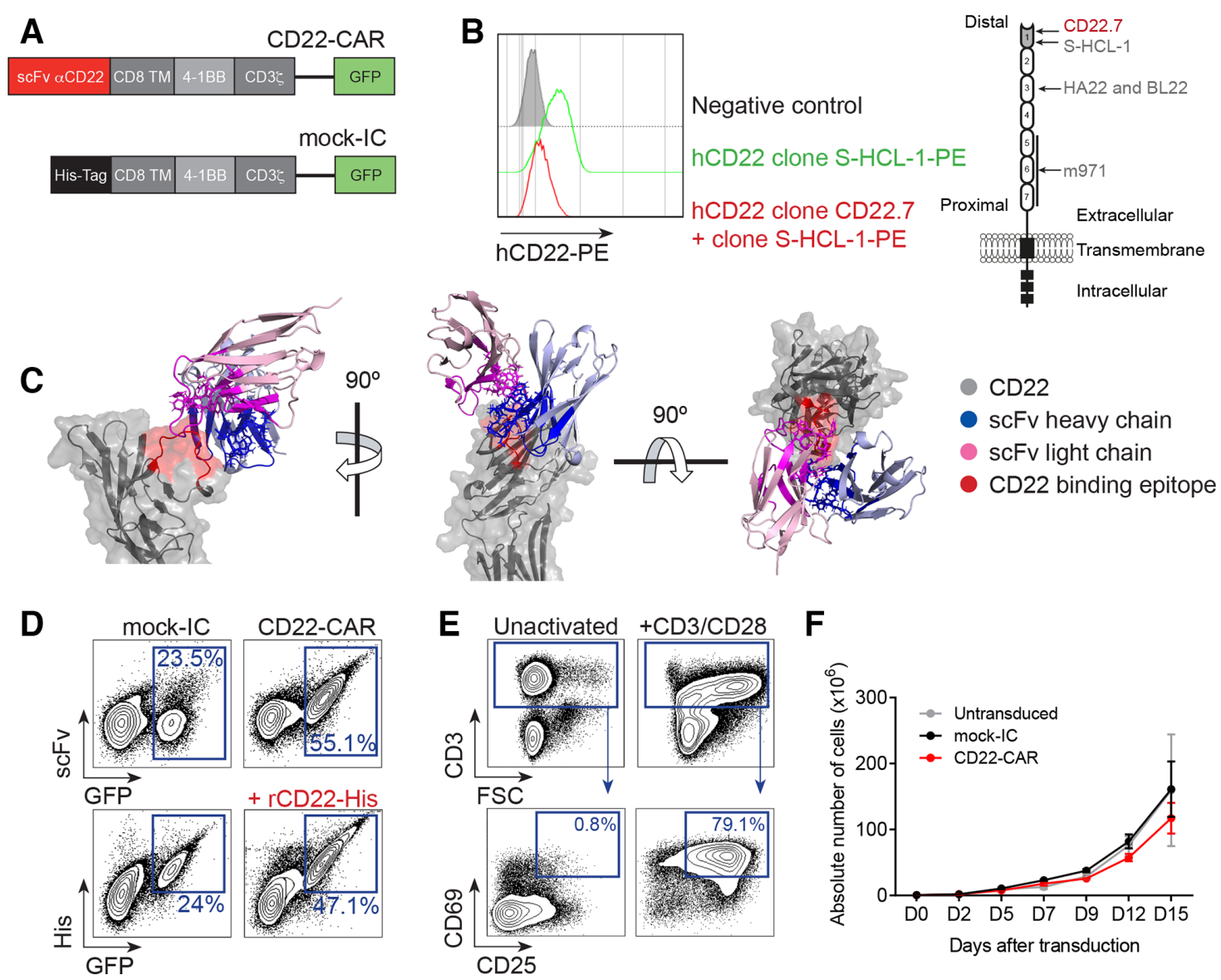

Figure 1 Design, detection and expansion of hCD22.7-CAR T cells. (A) Scheme of the hCD22.7-CAR structure and the mockIC. (B) Histogram showing the blocking of the binding of the anti-CD22 S-HCL-1 clone (PE signal) when CD22 ${ }^{+}$B cell line Raji cells are preincubated with the hCD22.7 clone (left panel). Right panel depicts the CD22 structure, indicating the binding site of hCD22.7 scFv (as well as m971, HA22 and BL22 scFvs). (C) Structural model of the CD22-hCD22.7-scFv complex. The structure is predicted based on the identified binding epitope of CD22 (ESTKDGKVP, in red) and the sequence of the heavy and light chains of the hCD22.7 scFv (blue and pink, respectively). (D) Representative CAR detection in primary T cells. CD22CAR-transduced T cells are detected as GFP and are corecognized by an anti-human IgG F(ab')2 and an anti-His moAb (on incubation with rCD22-His). (E) Proper T cell activation determined by CD25 and CD69 staining after 48 hours exposure to antiCD3/CD28 (PBMCs from $n=3$ healthy donors). ( $F$ ) Robust expansion of activated $T$ cells untransduced or transduced with either mock-IC or CD22-CAR (PBMCs from $n=3$ healthy donors). Plot shows mean \pm SEM ( $n=3$ healthy donors). CAR, CD22-chimeric antigen receptor; FSC, forward scatter; His, histidine; mock-IC, mock-intracellular; PBMCs, peripheral blood mononuclear cells; rCD22-His, recombinant CD22-His; scFv, single-chain variable fragment; TM, transmembrane. 

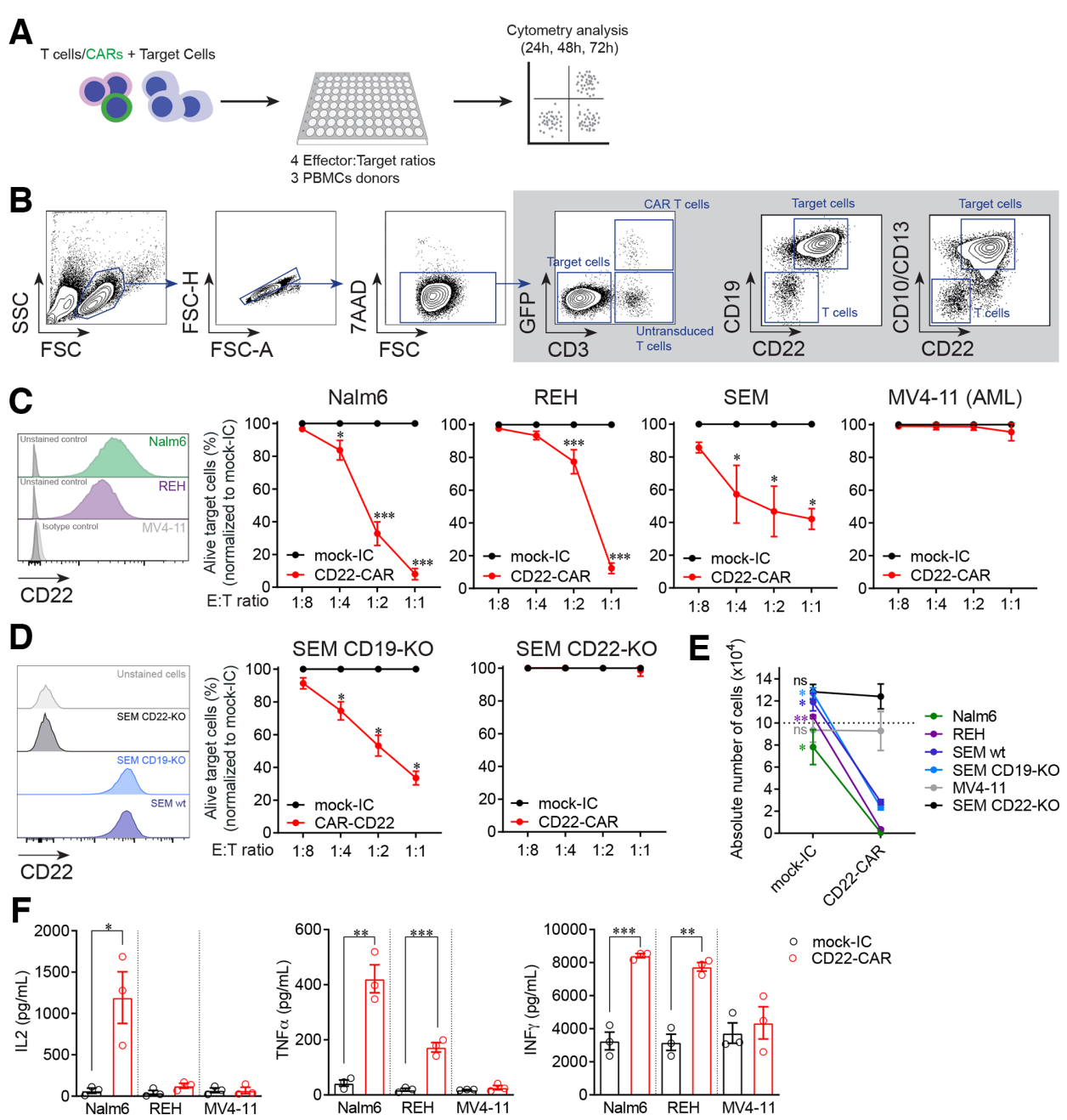

$\circ$ mock-IC
$\circ$ CD22-CAR
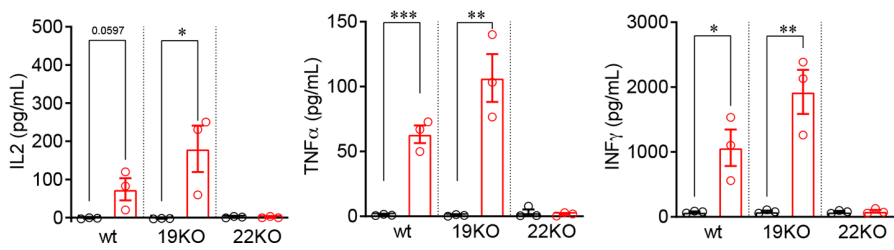

Figure 2 CD22-CAR T cells specifically target and eliminate B-ALL cell lines in vitro. (A) Experimental design of the in vitro cytotoxicity assays. (B) Flow cytometry gating strategy was used to analyze cytotoxicity. Alive target cells were identified as $7 \mathrm{AAD}^{-} \mathrm{CD} 3^{-} \mathrm{GFP}^{-} \mathrm{CD} 19^{+} \mathrm{CD} 10^{+} / \mathrm{CD} 13^{+}$(CD33 ${ }^{+}$for MV4-11 cell line). CD22 was not used to avoid confounding detection of target cells due to potential antigen loss. Doublets were removed from the analysis. (C) Percentage of alive target cells after 48 hours incubation with CD22-CAR T cells at the indicated E:T ratios. Results are normalized respect to mock-IC data (PBMCs from $\mathrm{n}=3$ healthy donors). CD22 expression in the different cell lines is shown previously in a histogram. (D) Specificity of CD22-CAR using CRISPR-Cas9 generated CD19-KO and CD22-KO SEM cells (PBMCs from $n=3$ healthy donors). CD22 expression in the different cell lines is shown previously in a histogram. (E) Absolute counts of alive target cells measured by FACS in 48 hours cytotoxicity assays at 1:1 $\mathrm{E}: \mathrm{T}$ ratio (PBMCs from $\mathrm{n}=3$ healthy donors). (F) ELISA showing robust secretion of proinflammatory cytokines by CD22-CAR T cells after 48 hours exposure to B-ALL and acute myeloid leukemia (AML) cell lines (upper panels) and wt, CD19-KO or CD22-KO SEM cell lines (bottom panels, PBMCs from $n=3$ healthy donors). Data are presented as mean \pm SEM ( $n=3$ healthy donors). ${ }^{*} p<0.05,{ }^{* *} p<0.01,{ }^{* * *} p<0.001$. B-ALL, B-cell acute lymphoblastic leukemia; CAR, CD22chimeric antigen receptor; E:T, effector:target ratio; FACS, fluorescence-activated cell sorting; FSC, forward scatter; FSC-A, forward scatter-area; FSC-H, forward scatter-high; mock-IC, mock intracellular; PBMCs, peripheral blood mononuclear cells; SSC, side scatter; KO, knock-out; MFI, median fluorescence intensity; wt, wild-type.

by the hCD22.7 moAb, we performed a cross-blocking assay with the anti-CD22 clone H-SCL-1, known to bind to the most distal Ig extracellular domain 1 of CD22. ${ }^{25}$ Binding of the labeled H-SCL-1 clone was hindered in cells preincubated with the antibody hCD22.7, indicating an overlapping binding of both moAbs to the most distal Ig domain of CD22 (figure 1B). Additionally, conformational epitope mapping assay, performed to exactly identify the sequence recognized by the hCD22.7 scFv, revealed that hCD22.7 scFv 
specifically binds to the ESTKDGKVP sequence, located in the Ig-like V-type domain, the most distal domain of CD22, thus confirming results from the cross-blocking assay (online supplementary figure 2). The molecular docking of this binding was computationally modeled (figure 1C and online supplementary file 1). The structural model inferred a CD22-hCD22.7-scFv complex energy of -1407.691 Rosetta score ${ }^{36}$ and a binding free energy of $-16.5615 \mathrm{kcal} / \mathrm{mol}$, using the InterfaceAnalyzer. ${ }^{38}$ These binding affinity parameters were comparable, or even slightly higher than those of the clinically approved high-affinity anti-CD22 moAb Epratuzumab $(-1005.624$ Rosetta score and $-11.345 \mathrm{kcal} / \mathrm{mol}$, respectively). ${ }^{30}$

Healthy human $\mathrm{T}$ cells were successfully transduced with both CD22-CAR and mock-IC lentivectors with a transduction efficiency of $20 \%-50 \%$. The expression of the CD22-CAR in T cells was confirmed by codetection of scFv and GFP, and by using an anti-His moAb on incubation with human rCD22-His (figure 1D). Importantly, activated $\left(\mathrm{CD} 69^{+} \mathrm{CD} 25^{+}\right) \mathrm{CD} 22-\mathrm{CAR}$ T cells (figure 1E) continuously expanded $>100$-fold over a 15-day period, similar to mock-IC $\mathrm{T}$ cells (figure $1 \mathrm{~F}$ ), demonstrating that hCD22.7-CAR expression does not hamper $\mathrm{T}$ cell expansion.

CD22-CAR T cells from three different healthy donors were then functionally tested in vitro in cytotoxicity assays against the B-ALL cell lines NALM6, SEM and REH, and the AML cell line MV4-11 as a negative control, at increasing E:T ratios (figure 2A). Alive target cells after hCD22.7-CAR $\mathrm{T}$ cell exposure were identified as 7AAD ${ }^{-}$ $\mathrm{CD}^{-} \mathrm{CD} 19^{+} \mathrm{CD}_{10} 0^{+}$(or $\mathrm{CD}^{+} 3^{+}$in the case of the SEM cells and $7 \mathrm{AAD}^{-} \mathrm{CD}^{-} \mathrm{CD}^{-} 3^{+}$in the case of MV4-11 cells, figure 2B). Compared with mock-IC T cells, hCD22.7-CAR $\mathrm{T}$ cells specifically eliminated CD22 ${ }^{+}$B-ALL cells, even at relatively low E:T ratios (figure 2C). Specificity of the hCD22.7-CAR $\mathrm{T}$ cells was further demonstrated using CRISPR/Cas9-mediated CD19-KO and CD22-KO SEM cells (figure 2D). Furthermore, B-ALL cells barely survived exposure to hCD22.7-CAR $\mathrm{T}$ cells in a 48 hours absolute number assay at a 1:1 E:T ratio (figure 2E). hCD22.7-CAR $\mathrm{T}$ cells produced high levels of the proinflammatory cytokines IL2, TNF $\alpha$, and IFN $\gamma$ after coculture with target cells, confirming their cytotoxicity (figure $2 \mathrm{~F}$ ).

\section{The expression level of CD22 impacts the in vitro efficiency of hCD22.7-CAR T cells in killing B-ALL primary cells}

It has been reported that remissions post-CD22-CAR T cell therapy are short and are associated with reduced expression of CD22, whereas high expression of CD22 favors the activity of CD22-CAR T cells. ${ }^{22}$ Moreover, whether $\mathrm{CD} 22^{-1}$ dim B-ALL relapses are common after CD22-CAR T cell treatment remains controversial. ${ }^{1521}$ Given this information, we next tested the efficacy of our CD22-CAR T cells in vitro, in nine primary B-ALL samples with variable expression levels of CD22. CD22 median fluorescence intensity (MFI) levels above or below the mean value $(\mathrm{MFI}=6752)$ were used to categorize primary B-ALLs as CD22 ${ }^{\text {high }}$ or $\mathrm{CD}_{2} 2^{\text {low }}$ (figure $3 \mathrm{~A}$ and online supplementary figure $3 \mathrm{a}$ ).
Cytotoxicity was measured after 24 hours and 48 hours exposure to CD22-CAR/mock-IC T cells (figure 3B and online supplementary figure 3b). Overall, hCD22.7-CAR $\mathrm{T}$ cells significantly eliminated primary B-ALL blasts (figure 3C). However, while a sustained cell elimination of $\mathrm{CD} 22^{\text {high }}$ B-ALL blasts $(\mathrm{n}=3)$ by hCD22.7-CAR $\mathrm{T}$ cells was consistently observed over a 48 hours period (figure 3C, left panel), hCD22.7-CAR T cell-mediated killing of $\mathrm{CD} 22^{\text {low }}$ B-ALL blasts $(\mathrm{n}=6)$, although significant, was less robust and occurred in a shorter or delayed cytotoxic window (figure 3C, right panel). Nevertheless, a similar trend of robust production of the proinflammatory cytokines IL2, TNF $\alpha$, and IFN $\gamma$ was observed for all B-ALL primary samples, regardless the expression levels of CD22 (figure 3D), confirming an efficient CD22 recognition and killing of B-ALL primary cells by our hCD22.7-CAR T cells.

\section{Membrane-distal CD22 epitope-directed CAR T cells efficiently eliminate B-ALL blasts in vivo in clinically relevant patient- derived xenografts}

We next assessed the activity of our hCD22.7-CAR in vivo using both CD22 $2^{\text {high }}$ (ALL\#1 and ALL\#2) and CD22 $2^{\text {low }}$ (ALL\#10) PDX B-ALL samples (figure 4, online supplementary table 1). NSG mice were intra-BM transplanted with $0.5-1.5 \times 10^{6}$ primary CD22 ${ }^{+}$B-ALL blasts, followed by infusion of $4 \times 10^{6} \mathrm{CD} 22-\mathrm{CAR} /$ mock-IC T cells 2 weeks later. Leukemic engraftment and $\mathrm{T}$ cell persistence were monitored by FACS biweekly in PB, and analyzed in $\mathrm{BM}$ and spleen at sacrifice, when any animal developed signs of overt disease (figure 4A,B). Of note, the kinetics of leukemia reconstitution (aggressiveness) detectable between week 4 and 20 post-transplant was unique for each $\mathrm{PDX}$, and the engraftment of both $\mathrm{CD} 22^{\text {high }}$ and $\mathrm{CD} 22^{\text {low }}$ PDXs tended progressively to increase over time in mock-IC $\mathrm{T}$ cell-treated mice (figure 4C). Importantly, however, hCD22.7-CAR T cells eradicated (ALL\#1 and ALL\#10) or largely controlled (ALL\#2) the growth of the both CD22 $2^{\text {high }}$ and CD22 $2^{\text {low }}$ B-ALL cells (figure 4C,D). T cell persistence adapted to the reconstitution kinetics of each PDX, and was detected in all three PDXs, even 26 weeks after CAR T cell infusion (range, week $6(n=15)$ to week $26(n=5)$ : mock-IC group $2.38 \% \pm 0.65 \%-0.34 \% \pm 0.34 \%$; CAR T cell group $2.79 \% \pm 1.13 \%-0.14 \% \pm 0.09 \%$, Figure $4 \mathrm{E}$ and online supplementary figure 4a-b). Disease control was further confirmed at sacrifice in ipsilateral (injected tibia) and contralateral (both femurs and non-injected tibia) BM, PB and spleen through FACS quantification of tumor burden (figure 4F and online supplementary figure 4c), lack of splenomegaly (figure 4G), and signs of hematopoietic displacement (figure 4H). Importantly, persistent leukemic cells at sacrifice maintained expression of CD22, likely ruling out CAR pressure-mediated antigen loss (figure 4I).

Finally, any engineered $\mathrm{scFv}$ of either murine or human origin expressed in autologous T cells may be immunogenic if presented on MHC-I, because peptides derived from it may be recognized as 'non-self' antigens by the adaptive immune system. ${ }^{39}$ We have thus compared the immunogenicity capacity of our hCD22.7-scFv side-by-side with that of 


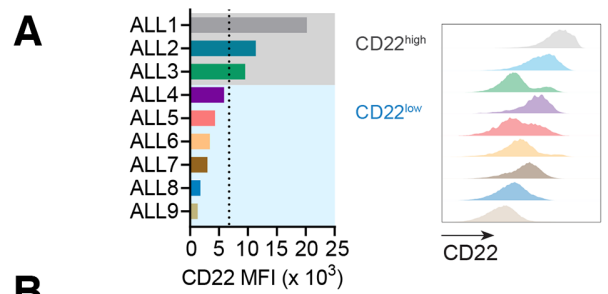
$\mathrm{CD} 22 \mathrm{MFI}\left(\times 10^{3}\right)$
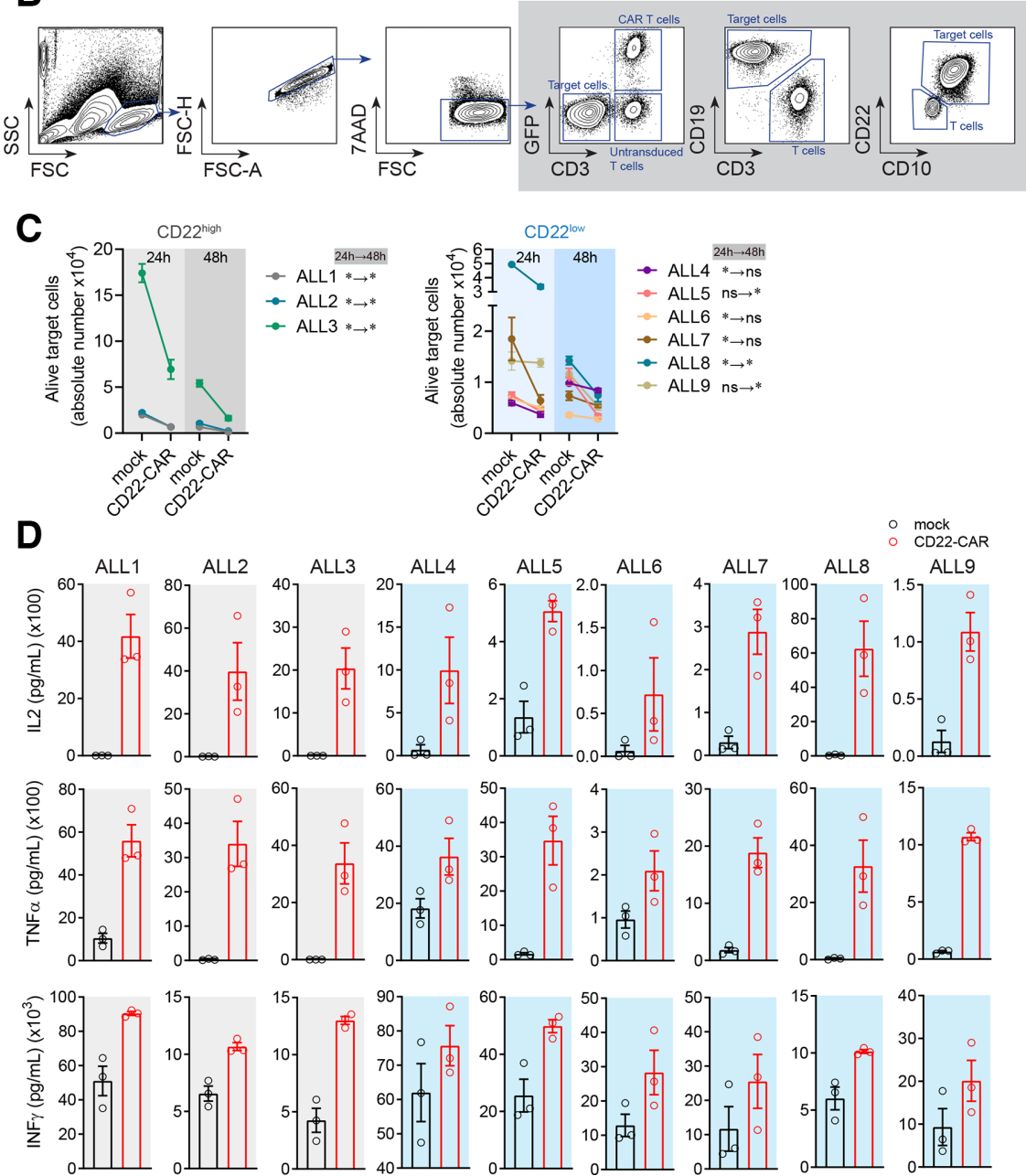

Figure 3 CD22-CAR T cells efficiently target and eliminate primary B-ALL cells in vitro although the expression level of CD22 impacts their efficacy. (A) MFI of CD22 in the different primary B-ALL samples used $(n=9)$. The mean value for CD22 MFI was used to classify primary B-ALL samples as CD22 high (ALL \#1 to ALL \#3) or CD22 ${ }^{\text {low }}$ (ALL \#4 to ALL \#9). (B) Flow cytometry gating strategy was used to analyze cytotoxicity. Alive target cells were identified as $7 A A D^{-} C D 3^{-} G_{F P}{ }^{-} C D 19^{+} C D 10^{+}$. CD22 was not used to avoid confounding detection of target cells due to potential antigen loss. Doublets were removed from the analysis. (C) Absolute counts of alive target CD22 high and CD22 ${ }^{\text {low }}$ cells measured by FACS in 24 hours and 48 hours cytotoxicity assays at 1:1 E:T ratio. The statistical significance is indicated for each sample. A two-way ANOVA test (Sidak's multiple comparison test) was used. (D) ELISA showing a similar robust production of IL2, TNF $\alpha$, and IFN $\gamma$ by CD22-CAR T cells after 48 hours exposure, among all B-ALL primary samples regardless the expression levels of CD22. Data are presented as mean \pm SEM (PBMCs from $n=3$ healthy donors). ${ }^{*} \mathrm{p}<0.05,{ }^{* \star} \mathrm{p}<0.01$. ANOVA, analysis of variance; $\mathrm{B}-\mathrm{ALL}$, B-cell acute lymphoblastic leukemia; CAR, CD22-chimeric antigen receptor; E:T, effector:target ratio; FACS, fluorescence-activated cell sorting; MFI, median fluorescence intensity; FSC, forward scatter; FSC-A, forward scatter-area; FSC-H, forward scatter-high; PBMCs, peripheral blood mononuclear cells; SSC, side scatter.

$\mathrm{m} 971^{15}$ and HA22 ${ }^{20}$ clones using the in silico tool NetMHC V.4.0 which predicts the affinity of peptides to bind to the most representative HLA supertypes. As shown in figure 5, we found very similar numbers of total (29, 23 and 26) and strong (8, 9 and 6) binding 9-mer peptides for hCD22.7, m971 and HA22, respectively.

\section{DISCUSSION/CONCLUSIONS}

CD19-CAR $\mathrm{T}$ cell therapy has shown indisputable potential in $\mathrm{R} / \mathrm{R}$ B-ALL. ${ }^{9} 10$ Unfortunately, however, $40 \%-60 \%$ of patients ultimately relapse because of poor CAR $\mathrm{T}$ cell persistence or the emergence of CD19B-ALL clones. ${ }^{11} 12$ Very few therapeutic options exist for 


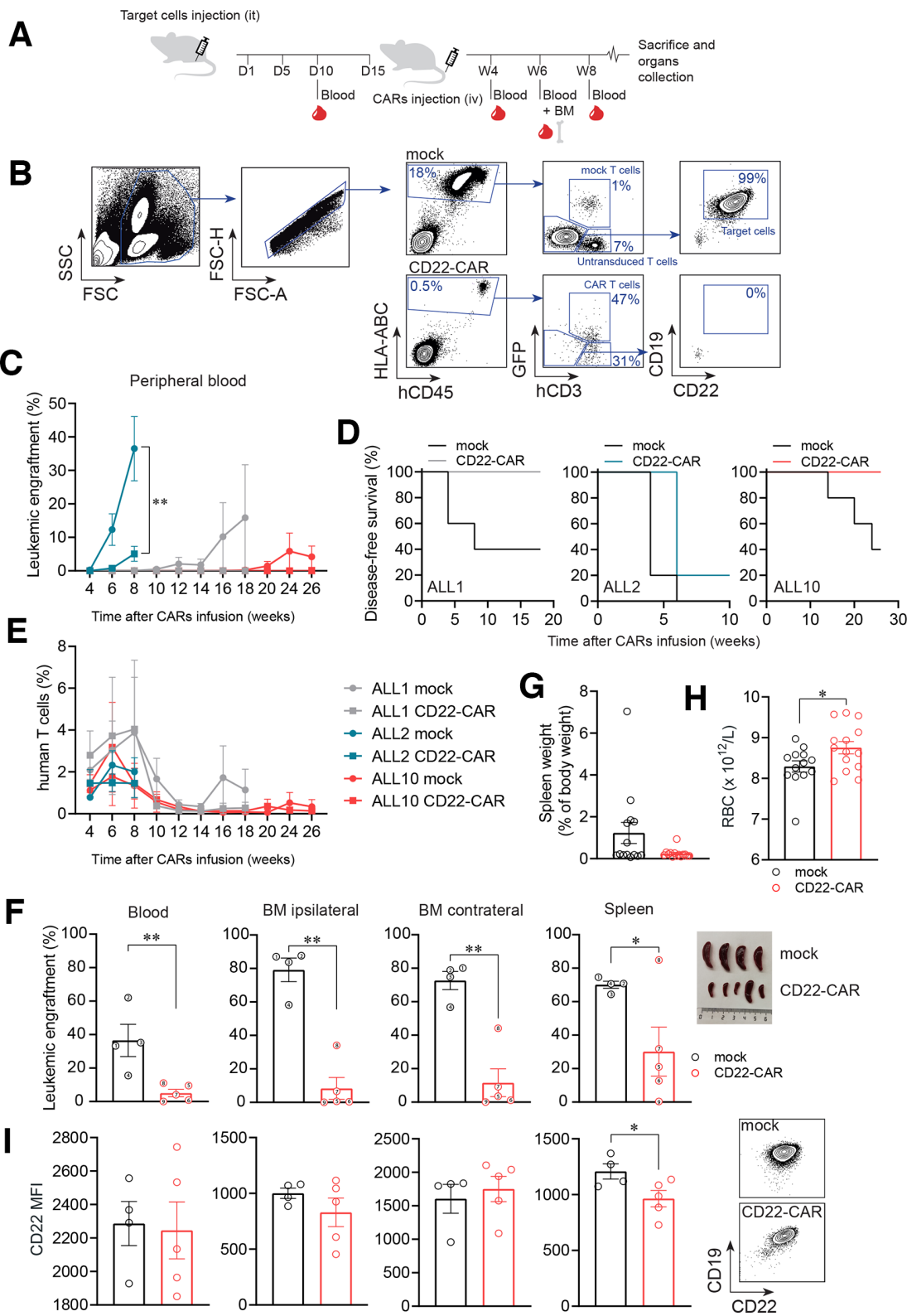

Figure 4 CAR T cells directed against a distal membrane CD22 epitope efficiently eliminate differentially aggressive PDX BALL blasts in vivo. (A) Experimental design of the in vivo experiments. (B) Gating strategy used to analyze FACS data from the in vivo experiments. Leukemic cells were identified as $h C D 45^{+} h H L A-A B C^{+} h C D 3^{-} h C D 19^{+} h C D 10^{+}$. CD22 was excluded from the identification to avoid confounding analysis due to potential antigen loss. CAR T cells were identified based on CD3 and GFP expression. Doublets were removed from the analysis. (C) Leukemic burden measured in PB over a 26 week follow-up period ( $\mathrm{n}=4-5$ mice/group; $\mathrm{n}=3$ PDXs, CD22 $2^{\text {high }}$ (ALL\#1 and ALL\#2) and CD22 ${ }^{\text {low }}$ (ALL\#10)). (D) Disease-free survival curves of mice transplanted with the three patient derived xenograft (PDXs). A mouse was considered 'with disease' when presenting $>0.1 \%$ blasts in PB. (E) T cell persistence measured in PB of mice transplanted with the three PDXs. (F) Leukemic burden at sacrifice in PB, ipsilateral and contralateral BM and spleen ( $n=9$ mice from the ALL\#2 PDX). Each mouse is identified with the same number across the plots. Macroscopic images of these spleens are also shown. (G) Spleen weight (total animals, $n=14$ ) from mock-IC and CD22-CAR T cell-treated mice at sacrifice. (H) PB RBC counts in mock-IC and CD22-CAR T cell-treated mice at sacrifice $(n=14)$. (I) CD22 MFI of persistent primary B-ALL in mock-IC-treated and CD22-CAR T cell-treated mice at sacrifice (ALL\#2 mice, $n=4-5$ ) and representative FACS plots of contralateral BM samples showing the levels of CD22 of a mock-IC and a CD22CAR T cell-treated mouse within the target cell population. Plot shows mean $\pm S E M$. ${ }^{*} p<0.05,{ }^{* \star} p<0.01,{ }^{* \star *} p<0.001$. B-ALL, B-cell acute lymphoblastic leukemia; BM, bone marrow; CAR, CD22-chimeric antigen receptor; D, day; FACS, fluorescence-activated cell sorting; PB, peripheral blood; it, intratibia; iv, intravenous; MFI, median fluorescence intensity; mock-IC, mock intracellular; RBC, red blood cell; W, week.

CD19- B-ALL relapse, ${ }^{40} 41$ and novel strategies, including the development of new target antigens, are being explored to enhance CAR T cell function and persistence. ${ }^{1342}$ CD22 is a pan B-cell antigen, ${ }^{14}$ and is an attractive alternative to CD19 for CAR T cell therapy because it will target CD19relapses, which usually retain CD22 expression. 

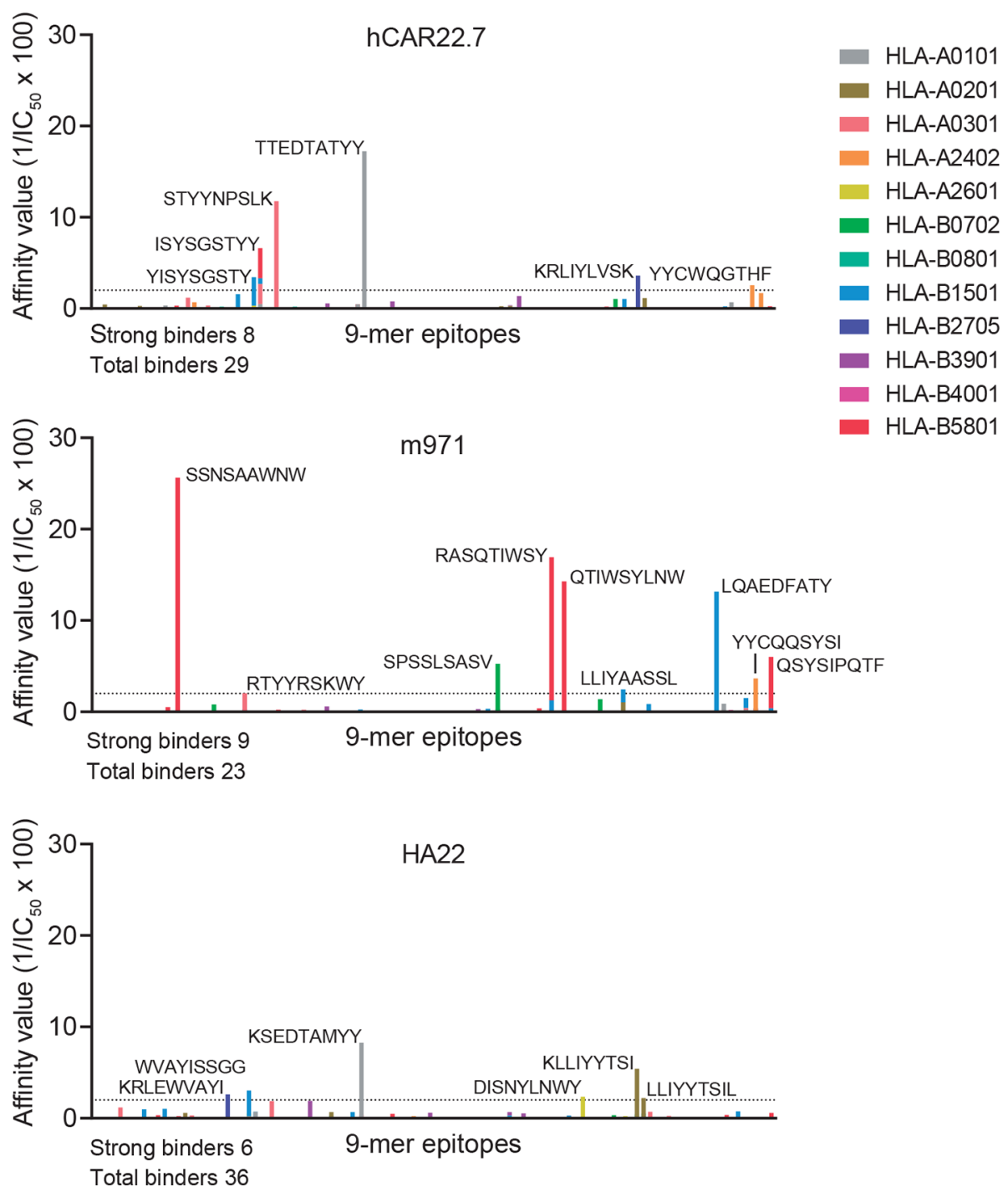

Figure 5 Immunogenic prediction of hCD22.7-scFv. The immunogenic potential of hCD22.7, m971 and HA22 CD22-scFvs was compared by in silico prediction analysis. CD22-scFvs were broken in 9-mer peptides, length for which human leukocyte antigen (HLA) molecules have a strong preference. Peptides are considered to bind $\mathrm{MHC}-\mathrm{I}$ when a IC $\mathrm{Is}_{50}<500 \mathrm{nM}$, being considered strong binders when $\mathrm{IC}_{50}<50 \mathrm{nM}$ (affinity value $>2$, dotted line) and amino acid sequence is shown in the figure. Only the most representative HLA supertypes were analyzed. The predicted epitope-binding affinity was calculated as $1 / \mathrm{IC}_{50} 100$. Predicted epitopes are distributed along the x-axis according to their distribution in the protein. $\mathrm{IC}_{50}$, half-maximum inhibitory concentration; scFv, single-chain variable fragment.

In this line, several CD22-CARs have been reported to induce clinical remission in $60 \%-70 \%$ of patients with $\mathrm{R} / \mathrm{R}$ B-ALL either naive or resistant to CD19-CAR T cells. ${ }^{15}$ 20-22 But, phase I clinical studies based on different CD22 scFvs (m971 vs YK-CD22BB-002) have reported preliminary results concerning CD22 loss and immune escape on CD22-CAR T cell treatment. ${ }^{15} 21$ A previous comprehensive comparison of several anti-CD22 moAbs indicated that epitope selection is crucial for the antileukemic activity of the CD22-CAR, and that the m971 scFv, which targets the most membrane-proximal Ig extracellular domains of CD22 (5 to 7), seems more potent than BL22 and HA22 scFvs, which target the proximal Ig domain $3{ }^{20}$ In this study, we report the generation and preclinical development of a new CD22-CAR that contributes to the limited state-of-the-art arsenal of CD22-CARs. Three CD22 reactive hybridomas were generated using conventional moAb generation techniques, rendering three very specific IgG1 isotype anti-CD22 moAbs, finally selecting the hCD22.7 clone for the construction of the CAR. To the best of our knowledge, this $a b$ initio generated hCD22.7 scFv is the first used for the development of a CD22-CAR recognizing the most membrane-distal Ig extracellular domain 1 of CD22. Additionally, we provide an uncommon comprehensive characterization including the molecular docking, epitope mapping, binding affinity, and immunogenicity of the hCD22.7 scFv.

Previous studies have addressed the impact of antigen density on CD22-CAR T cell efficacy using a higher-affinity version of the $\mathrm{m} 971 \mathrm{scFv}$, and reported a positive correlation between CD22 expression and the functionality of CD22-CAR $T$ cells, both in vitro and in vivo, using cell 
lines and one patient derived xenograft $(\mathrm{PDX}) .^{22}$ Here, the expression level of CD22 was used to classify primary B-ALL samples as CD22 $2^{\text {high }}$ or $\mathrm{CD} 22^{\text {low }}$, and we show that although our high-affinity hCD22.7-CAR efficiently and consistently targeted $\mathrm{CD}_{22}{ }^{+}$cells, it displayed a differential killing kinetics depending on the expression level of CD22. While a sustained cell elimination of CD22 $2^{\text {high }}$ cells was observed over a 48 hours period, a shorter or delayed but still efficient cytotoxic window was observed for CD22 ${ }^{\text {low }}$ cells. It is also plausible that CD22 adopts different conformational epitope exposures ${ }^{43}$ affecting the performance of the CAR $\mathrm{T}$ cells in the different samples. Of note, a robust production of proinflammatory cytokines was observed for all B-ALL primary samples, regardless the expression levels of CD22, confirming an efficient CD22 recognition and killing of B-ALL primary cells by our hCD22.7-CAR T cells.

Our membrane distal epitope hCD22.7-CAR T cells performed competently in controlling in vivo several B-ALL PDXs with varied aggressiveness for a long period, which was coupled to long-term $\mathrm{T}$ cell persistence. In fact, hCD22.7-CAR T cells were capable of eradicating longterm disease in several PDXs, with persistence of T cells even after 26 weeks. In the PDX ALL\#2, although the leukemia burden was not fully eradicated, it was significantly controlled. The not complete eradication of this PDX may reflect a more aggressive molecular subtype, a superior intrinsic refractoriness due to resistance generated through multiple lines of previous treatments, a faster/deeper graft of this particular PDX, a worse pharmacodynamics of CAR $\mathrm{T}$ cells in this particular case perhaps due to peripheral filtration, and so on. Of note, we found no apparent signs of CD22 antigen loss by the few surviving/resistant B-ALL cells in vivo. Antigen loss represents one non-exclusive potential mechanism of immune escape and largely relies on tumor-specific cellautonomous properties, differentiation stage in which leukemic cells are stalled, and the complexity of immune cellular and soluble interactions, difficult to reconstruct in xenograft models. In addition, it cannot be ruled out that residual CAR-resistant $\mathrm{CD}_{2}{ }^{+}$leukemic cells have simply not been encountered by CD22-CAR T cells. Only a controlled and homogeneous phase I clinical trial will reliably inform about a potential target antigen loss and immune scape. Technically, our experimental design used a rigorous 'mock' control where all the structural, cytolytic and costimulatory motifs are expressed in the effector $\mathrm{T}$ cells, but without the extracellular anti-CD22 $\mathrm{scFv}$ region. This intracellular mock further validates the specificity and sensitivity of our hCD22.7-CAR.

Our hCD22.7-derived membrane distal-targeting CAR has not been functionally compared side-by-side with other membrane proximal-targeting CD22-scFvs used already in clinical trials, ${ }^{1521}$ with which Fry et al published discrete complete remission (CR) rates of $57 \%$ with a median remission duration of 6 moths in patients with $\mathrm{R} / \mathrm{R}$ B-ALL treated with m971-CD22-CAR T cells ${ }^{15}$ and in a more recent study they reported CR of $70 \%$ with a median relapse-free survival of 6 months. ${ }^{23}$ However, the hCD22.7-CAR performs efficiently in vitro and in vivo using clinically relevant patient samples of B-ALL with different aggressiveness. This coupled to the long-term persistence of hCD22.7-CAR T cells, the lack of antigenic loss in vivo in the few CAR-resistant B-ALL cells, its high affinity, and its immunogenicity similar to m971 and HA22 CD22-scFvs provides support for its clinical validation as either single or tandem CD22-CD19-CAR for both naive and anti-CD19-resistant patients with B-ALL.

\section{Author affiliations}

${ }^{1}$ Josep Carreras Leukemia Research Institute, Barcelona, Spain

${ }^{2}$ Sección de Oncohematología Pediátrica, Hospital Clínico Universitario Virgen de la Arrixaca and Instituto Murciano de Investigación Biosanitaria (IMIB), El Palmar, Murcia, Spain

${ }^{3}$ Department of Pediatric Hemato-oncology, Armand-Trousseau Childrens Hospital, Paris, Île-de-France, France

${ }^{4}$ Centro de Investigación Biomédica en Red de Cáncer (CIBER-ONC), Instituto de Salud Carlos III, Barcelona, Spain

${ }^{5}$ Department of Biosciences, Universitat de Vic - Universitat Central de Catalunya, Vic, Catalunya, Spain

${ }^{6}$ Institut d'Investigacions Biomèdiques, August Pi i Sunyer, Barcelona, Spain ${ }^{7}$ Department of Biomedicine, School of Medicine, University of Barcelona, Barcelona, Spain

${ }^{8}$ Instituciò Catalana de Recerca I Estudis Avançats (ICREA), Barcelona, Spain

Acknowledgements We thank CERCA/Generalitat de Catalunya and Fundació Josep Carreras-0bra Social la Caixa for their institutional support.

Contributors TVH conceived the study, designed and performed experiments, analyzed data, and wrote the manuscript. SRZ designed and performed experiments. HRH, FGA, PP, MLB, OM, and DSM performed experiments. NFF generated the structural model. PE generated the CD22 hybridoma and performed experiments. JLF and PB provided primary samples. CB supported the study technically and financially. PM conceived the study, designed experiments, wrote the manuscript, and financially supported the study.

Funding This work was supported by the European Research Council (CoG-2014646903, PoC-2018-811220), the Spanish Ministry of Economy and Competitiveness (MINECO-SAF2016-80481-R), the Fundación Uno entre Cienmil, the Obra Social La Caixa (LCF/PR/HR19/52160011), the Spanish Cancer Association and Leo Messi Foundation to PM. CB was supported by the ISCIII/FEDER (PI17/01028). TVH and SRZ were supported by Marie Sklodowska Curie Fellowships (792923 and 795833, respectively). DSM was supported by a Sara Borrell fellowship from the ISCIII. MLB was supported by a FPI scholarship from the MINECO. PM is an investigator of the Spanish Cell Therapy Network (TERCEL).

Competing interests PM is co-founder of OneChain Immunotherapeutics $(\mathrm{OCI})$, a IJC spin-off company focused on the development of CAR T-cell therapies. The patent of the membrane-distal CD22 scFv clone has been licenced to $0 \mathrm{Cl}$.

\section{Patient consent for publication Not required.}

Provenance and peer review Not commissioned; externally peer reviewed.

Data availability statement Data sharing not applicable as no datasets generated and/or analyzed for this study. Data are available upon reasonable request. All data relevant to the study are included in the article or uploaded as supplementary information.

Open access This is an open access article distributed in accordance with the Creative Commons Attribution Non Commercial (CC BY-NC 4.0) license, which permits others to distribute, remix, adapt, build upon this work noncommercially, and license their derivative works on different terms, provided the original work is properly cited, appropriate credit is given, any changes made indicated, and the use is non-commercial. See http://creativecommons.org/ licenses/by-nc/4.0/.

\section{ORCID iDs}

Talia Velasco-Hernandez http://orcid.org/0000-0003-2183-7443

Samanta Romina Zanetti http://orcid.org/0000-0002-9052-8751

Pablo Engel http://orcid.org/0000-0001-8410-252X 


\section{REFERENCES}

1 Greaves M. A causal mechanism for childhood acute lymphoblastic leukaemia. Nat Rev Cancer 2018;18:471-84.

2 Smith MA, Altekruse SF, Adamson PC, et al. Declining childhood and adolescent cancer mortality. Cancer 2014;120:2497-506.

3 Pui C-H, Yang JJ, Hunger SP, et al. Childhood acute lymphoblastic leukemia: progress through collaboration. J Clin Oncol 2015;33:2938-48.

4 Bhojwani D, Pui C-H. Relapsed childhood acute lymphoblastic leukaemia. Lancet Oncol 2013;14:e205-17.

5 Schrappe M, Hunger SP, Pui C-H, et al. Outcomes after induction failure in childhood acute lymphoblastic leukemia. N Engl J Med 2012;366:1371-81.

6 Sellar RS, Rowntree C, Vora AJ, et al. Relapse in teenage and young adult patients treated on a paediatric minimal residual disease stratified all treatment protocol is associated with a poor outcome: results from UKALL2003. Br J Haematol 2018;181:515-22.

7 Sun W, Malvar J, Sposto R, et al. Outcome of children with multiply relapsed $B$-cell acute lymphoblastic leukemia: a therapeutic advances in childhood leukemia \& lymphoma study. Leukemia 2018;32:2316-25.

8 Faderl S, O'Brien S, Pui C-H, et al. Adult acute lymphoblastic leukemia: concepts and strategies. Cancer 2010;116:1165-76.

9 Davila ML, Riviere I, Wang X, et al. Efficacy and toxicity management of 19-28z CAR T cell therapy in B cell acute lymphoblastic leukemia. Sci Transl Med 2014;6:224ra25.

10 Maude SL, Frey N, Shaw PA, et al. Chimeric antigen receptor $\mathrm{T}$ cells for sustained remissions in leukemia. $N$ Engl J Med 2014;371:1507-17.

11 Sotillo E, Barrett DM, Black KL, et al. Convergence of acquired mutations and alternative splicing of CD19 enables resistance to CART-19 immunotherapy. Cancer Discov 2015;5:1282-95.

12 Ghorashian S, Kramer AM, Onuoha S, et al. Enhanced CAR T cell expansion and prolonged persistence in pediatric patients with all treated with a low-affinity CD19 CAR. Nat Med 2019;25:1408-14.

13 Labanieh L, Majzner RG, Mackall CL. Programming CAR-T cells to kill cancer. Nat Biomed Eng 2018;2:377-91.

14 Nitschke L. Cd22 and Siglec-G: B-cell inhibitory receptors with distinct functions. Immunol Rev 2009;230:128-43.

15 Fry TJ, Shah NN, Orentas RJ, et al. CD22-targeted CAR T cells induce remission in B-ALL that is naive or resistant to CD19-targeted CAR immunotherapy. Nat Med 2018;24:20-8.

16 van Zelm MC, van der Burg M, de Ridder D, et al. Ig gene rearrangement steps are initiated in early human precursor $B$ cell subsets and correlate with specific transcription factor expression. $J$ Immunol 2005;175:5912-22.

17 Kantarjian $\mathrm{H}$, Thomas D, Jorgensen J, et al. Results of inotuzumab ozogamicin, a CD22 monoclonal antibody, in refractory and relapsed acute lymphocytic leukemia. Cancer 2013;119:2728-36.

18 Mussai F, Campana D, Bhojwani D, et al. Cytotoxicity of the antiCD22 immunotoxin HA22 (CAT-8015) against paediatric acute lymphoblastic leukaemia. Br J Haematol 2010;150:352-8.

19 Wayne AS, Kreitman RJ, Findley HW, et al. Anti-CD22 immunotoxin RFB4(dsFv)-PE38 (BL22) for CD22-positive hematologic malignancies of childhood: preclinical studies and phase I clinica trial. Clin Cancer Res 2010;16:1894-903.

20 Haso W, Lee DW, Shah NN, et al. Anti-CD22-chimeric antigen receptors targeting $B$-cell precursor acute lymphoblastic leukemia. Blood 2013;121:1165-74.

21 Pan J, Niu Q, Deng B, et al. Cd22 CAR T-cell therapy in refractory or relapsed $B$ acute lymphoblastic leukemia. Leukemia 2019;33:2854-66.

22 Ramakrishna S, Highfill SL, Walsh Z, et al. Modulation of target antigen density improves CAR T-cell functionality and persistence. Clin Cancer Res 2019;25:5329-41.
23 Shah NN, Highfill SL, Shalabi H, et al. Cd4/Cd8 T-cell selection affects chimeric antigen receptor (CAR) T-cell potency and toxicity: updated results from a phase I anti-CD22 CAR T-cell trial. J Clin Oncol 2020;38:1938-50.

24 Abdiche YN, Yeung AY, Ni l, et al. Antibodies targeting closely adjacent or minimally overlapping epitopes can displace one another. PLoS One 2017;12:e0169535.

25 Engel P, Wagner N, Miller AS, et al. Identification of the ligandbinding domains of CD22, a member of the immunoglobulin superfamily that uniquely binds a sialic acid-dependent ligand. J Exp Med 1995;181:1581-6.

26 Andreatta M, Nielsen M. Gapped sequence alignment using artificial neural networks: application to the MHC class I system. Bioinformatics 2016;32:511-7.

27 Nielsen M, Lundegaard C, Worning P, et al. Reliable prediction of T-cell epitopes using neural networks with novel sequence representations. Protein Sci 2003;12:1007-17.

28 Gubin MM, Zhang X, Schuster H, et al. Checkpoint blockade cancer immunotherapy targets tumour-specific mutant antigens. Nature 2014;515:577-81.

29 Rose PW, Beran B, Bi C, et al. The RcsB protein data bank: redesigned web site and web services. Nucleic Acids Res 2011;39:D392-401.

30 Ereño-Orbea J, Sicard T, Cui H, et al. Molecular basis of human CD22 function and therapeutic targeting. Nat Commun 2017;8:764.

31 Fernandez-Fuentes N, Rai BK, Madrid-Aliste CJ, et al. Comparative protein structure modeling by combining multiple templates and optimizing sequence-to-structure alignments. Bioinformatics 2007;23:2558-65.

32 Tapryal S, Gaur V, Kaur KJ, et al. Structural evaluation of a mimicryrecognizing paratope: plasticity in antigen-antibody interactions manifests in molecular mimicry. J Immunol 2013;191:456-63.

33 Wright LM, Brzozowski AM, Hubbard RE, et al. Structure of Fab hGR-2 F6, a competitive antagonist of the glucagon receptor. Acta Crystallogr D Biol Crystallogr 2000;56:573-80.

34 Sugiyama K, lyori M, Sawaguchi A, et al. The crystal structure of the active domain of Anopheles anti-platelet protein, a powerful anti-coagulant, in complex with an antibody. J Biol Chem 2014;289:16303-12.

35 Schneidman-Duhovny D, Inbar Y, Nussinov R, et al. PatchDock and SymmDock: servers for rigid and symmetric docking. Nucleic Acids Res 2005;33:W363-7.

36 Leaver-Fay A, Tyka M, Lewis SM, et al. ROSETTA3: an objectoriented software suite for the simulation and design of macromolecules. Methods Enzymol 2011;487:545-74.

37 Sánchez-Martínez D, Baroni ML, Gutierrez-Agüera F, et al. Fratricideresistant CD1a-specific CAR T cells for the treatment of cortical T-cell acute lymphoblastic leukemia. Blood 2019;133:2291-304.

38 Stranges PB, Kuhlman B. A comparison of successful and failed protein interface designs highlights the challenges of designing buried hydrogen bonds. Protein Sci 2013;22:74-82.

39 Yadav M, Jhunjhunwala S, Phung QT, et al. Predicting immunogenic tumour mutations by combining mass spectrometry and exome sequencing. Nature 2014;515:572-6.

40 Aldoss I, Bargou RC, Nagorsen D, et al. Redirecting T cells to eradicate B-cell acute lymphoblastic leukemia: bispecific T-cell engagers and chimeric antigen receptors. Leukemia 2017;31:777-87.

41 Sikaria S, Aldoss I, Akhtari M. Monoclonal antibodies and immune therapies for adult precursor B-acute lymphoblastic leukemia. Immunol Lett 2016;172:113-23.

42 Raponi S, De Propris MS, Intoppa S, et al. Flow cytometric study of potential target antigens (CD19, CD20, CD22, CD33) for antibodybased immunotherapy in acute lymphoblastic leukemia: analysis of 552 cases. Leuk Lymphoma 2011;52:1098-107.

43 Xiao X, Ho M, Zhu Z, et al. Identification and characterization of fully human anti-CD22 monoclonal antibodies. MAbs 2009;1:297-303. 